MONTANA

STATE UNIVERSITY

LIBRARY

\title{
Deuteron Intrabond Motion and Ferroelectricity in $\mathrm{KD}_{2} \mathrm{PO}_{4}$
}

\section{Authors: Henry Silsbee, Edwin Uehling, and V. Hugo Schmidt}

This is a the published version of an article that originally appeared in Journal of Scientific Instruments in December 1965.

Silsbee, Henry, Edwin Uehling, and V. Schmidt. "Deuteron Intrabond Motion and Ferroelectricity in KD2PO4.” Physical Review 133, no. 1A (January 1964): A165-A170.

doi: $\underline{10.1103 / p h y s r e v .133 . a 165}$.

Made available through Montana State University's ScholarWorks

scholarworks.montana.edu 
ferent from the moments relevant to the no-phonon line alone..$^{1,3,11}$ The no-phonon line is therefore a "narrowed" line in the sense that its width is less than is predicted by the second moment of the complete line. ${ }^{12}$ In the physical picture of Bloembergen, Purcell, and Pound, implicit in Eq. (1), we would attribute this narrowing to the fact that very rapid perturbations in the energy separation $\hbar \Omega(t)$ "average out" in the time required for their measurement and do not contribute to the width of the no-phonon line. One should not infer from this, however, that the high-frequency perturbations do not modify the observed spectrum. It is

${ }^{11}$ A. Kiel in Advances in Quantum Electronics, edited by J. R. Singer (Columbia University Press, New ${ }_{\text {Hi }}$ York, 1961), p. 417; R. H. Silsbee, Phys. Rev. 128, 1726,(1962) and 129, 2835 (1963)

${ }_{12}$ J. H. Van Vleck, Phys. Rev. 55, 924 (1939); M. Lax, J. Chem. Phys. 20, 1752 (1952); M. Lax and E. Burstein, Phys. Rev. 100 592 (1955); H. Gummel and M. Lax, Ann. Phys. (Paris) 2, 28 (1957).

PHYSICAL REVIEW

VOLUME 133, NUMBER 1A

6 JANUARY 1964

\title{
Deuteron Intrabond Motion and Ferroelectricity in $\mathrm{KD}_{2} \mathrm{PO}_{4} \dagger$
}

\author{
Henry B. Silsbee and Edwin A. Uehling \\ University of Washington, Seattle, Washington \\ AND \\ V. Hugo Schmidt \\ Valparaiso University, Valparaiso, Indiana \\ (Received 26 August 1963)
}

\begin{abstract}
The Slater theory of the ferroelectric phase transition in $\mathrm{KH}_{2} \mathrm{PO}_{4}$ type crystals as modified by Takagi and extended by Senko is examined again in the light of recent measurements of parameters made on $\mathrm{KD}_{2} \mathrm{PO}_{4}$. Making full use of the measurements, and assigning an appropriate numerical value to one adjustaable parameter which is not independently measured, the theory gives a nearly correct description of the shape of the spontaneous polarization curve. Also the value obtained for the Curie constant is probably satisfactory in view of limitations of the model and uncertainty of the high-temperature experimental data. The theory fails, however, in at least one important respect; the transition entropy is overestimated. This and other discrepancies are discussed briefly.
\end{abstract}

\section{INTRODUCTION}

A POSSIBLE connection between the measured activation energy of deuterons jumping between two off-center equilibrium positions in the hydrogen bond of $\mathrm{KD}_{2} \mathrm{PO}_{4}$, and a particular model of the ferroelectric phase transition in these crystals has been noted. ${ }^{1}$ The model used is the one described by Slater ${ }^{2}$ and modified by Takagi, ${ }^{3}$ the principal feature of which is the short-range ordering of the hydrogen positions. The measured activation energy has been identified ${ }^{1}$ with the energy of one of the hydrogen configurations.

$\dagger$ Research supported by the National Science Foundation and by the U. S. Army Research Office Durham, South Carolina.

1 V. H. Schmidt and E. A. Uehling, Phys. Rev. 126, 477 (1962).

2 J. C. Slater, J. Chem. Phys. 9, 16 (1941).

3. Takagi, J. Phys. Soc. Japan 3, 271, 273 (1948).
An extension of the theory was subsequently made by Senko, ${ }^{4}$ who did not, however, make use of the measured activation energy as a basis for a numerical test of the theory. The essential feature of his modification is the introduction of long-range polarization forces. The result is a theory containing both longrange and short-range forces of certain specified types, some of which are capable of independent measurement.

It is the purpose of this paper to review this theory and to describe pertinent features of the experimental data which tend to provide a justification of it. As we show, all of the important experimental parameters can be unambiguously determined, in part by experiments which are not directly related to specific ferro-

\footnotetext{
${ }^{4}$ M. E. Senko, Phys. Rev. 121, 1599 (1961).
} 


\begin{tabular}{|c|c|c|c|}
\hline Configuration & Energy & Moment & $\begin{array}{l}\text { Fractional } \\
\text { Population }\end{array}$ \\
\hline$=20$ & 0 & $\mu_{0}$ & $x_{2}$ \\
\hline 1 ब & 0 & $-\mu_{0}$ & $x_{-2}$ \\
\hline 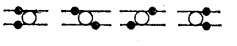 & $\epsilon_{0}$ & 0 & $x_{0}$ each \\
\hline कृ & $\epsilon_{1}$ & $\mu_{0} / 2$ & $x_{1}$ each \\
\hline 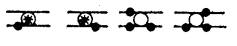 & $\epsilon_{1}$ & $-\mu_{0} / 2$ & $X_{-1}$ each \\
\hline$\rightarrow \infty$ & $\epsilon_{2}$ & 0 & $x_{4}$ each \\
\hline
\end{tabular}

FIG. 1. Schematic representation of $\mathrm{PO}_{4}$ ions and their close hydrogens.

electric properties, and in part by observations which are related to them. We show that both the shape of the spontaneous polarization curve and the magnitude of the Curie constant for the susceptibility are then nearly correctly given. What discrepancies or uncertainties remain are to be attributed, at least in part, to uncertainties in the experimental data. There is, however, one outstanding discrepancy; namely, the predicted value of the transition heat is almost a factor of two higher than the quoted experimental value.

A brief description of the model and of the conclusions about the nature of the ferroelectric phase transition which is predicted by it is given in Sec. II; a justification of some aspects of this model including a comparison with ferroelectric data and specific heat measurements is given in Sec. III; and finally, some suggestions with regard to the possible elimination of outstanding discrepancies are made in Sec. IV.

\section{MODEL}

The crystal has chains along the $z$ direction of alternating $\mathrm{K}$ atoms and $\mathrm{PO}_{4}$ ions. Adjacent chains are displaced along $z$ by $c / 4$ and are linked by $\mathrm{H}$ bonds connecting an $\mathrm{O}$ atom at the top of a $\mathrm{PO}_{4}$ group to one at the bottom of its neighbor. The $\mathrm{H}$ bonds are very nearly along the $x$ and $y$ directions. If the top of a $\mathrm{PO}_{4}$ group has " $x$ bonds", its bottom has " $y$ bonds."

The model supposes that at all temperatures there is an $\mathrm{H}$ in each bond and that it occupies one of two off-center positions, a "plus bond" having the $\mathrm{H}$ nearer the $\mathrm{O}$ which is at the top of its ion. Thus, there are various possible configurations of a $\mathrm{PO}_{4}$ ion and its near H's, if any, as indicated in Fig. 1.

The model assigns to each configuration a contribution to the energy, a contribution to the $z$ component of dipole moment, and a fractional population as indicated. We have anticipated some trivial results of the analysis by setting certain populations equal $a$ priori.

We suppose that the energy has the form

$$
U=\text { (short-range terms) }-V P\left(E_{\text {ext }}+B P\right) \text {. }
$$

where $P$ is the polarization, $E_{\text {ext }}=E+\alpha P$ is the field of external charges, $\alpha$ is the "depolarizing factor" appropriate to the specimen shape, and the last term represents the long-range Coulomb effect within the sample. We take

$$
P=\left(N \mu_{0} / V\right) p+\chi_{0} E \text { with } p=x_{2}-x_{-2}+2\left(x_{1}-x_{-1}\right) .
$$

The first term gives the explicit contributions associated with the hydrogen configurations of Fig. 1, while the second allows for any further induced contributions in perhaps the simplest of various possible ways. We then find

$$
U=N\left[4 x_{0} \epsilon_{0}+4\left(x_{1}+x_{-1}\right) \epsilon_{1}+2 x_{4} \epsilon_{2}-\mu_{1} E p-\beta p^{2}-\gamma E^{2}\right],
$$

where $\mu_{1}=\mu_{0}+\left(2 V \chi_{0} \beta / N \mu_{0}\right)$, and we regard $\beta$ (which may be shape dependent) as an adjustable parameter. The $E^{2}$ term plays no significant role and will be dropped.

The entropy is, by a slight generalization of Takagi's result,

$$
S=N k \ln \frac{b_{+}{ }^{2 b+} b_{-}{ }^{2 b-}}{x_{2}{ }^{x_{2}} x_{-2}{ }^{x-2} x_{0}{ }^{4 x_{0}} x_{1}{ }^{4 x_{1}} x_{-1}{ }^{4 x-1} x_{4}{ }^{2 x_{4}}}
$$

where $b_{+}=x_{2}+2 x_{0}+3 x_{1}+x_{-1}+x_{4}=\frac{1}{2}(1+p)$ is the fraction of "plus" bonds and similarly for $b_{-}$.

The free energy may be minimized with respect to the populations for a given $p$ to yield:

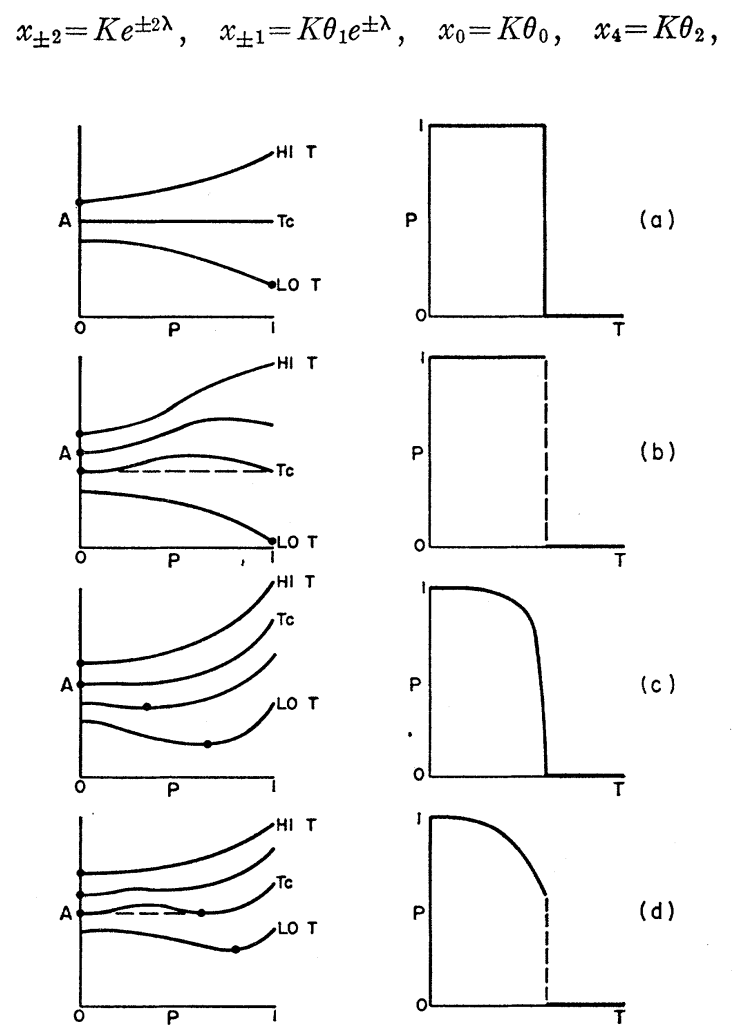

FIG. 2. Qualitative behavior of free energy versus polarization and spontaneous polarization versus temperature. The free energy curves have been displaced vertically to put the high-temperature curves at the top. 


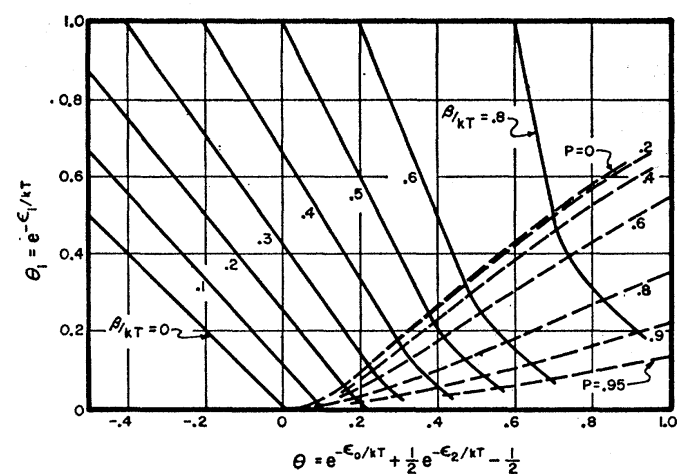

Fig. 3. Parameter combinations for which the phase transition occurs. $p$ is the fractional value to which the polarization jumps in the case of a first-order transition.

where

$$
\begin{gathered}
\theta_{i}=e^{-\epsilon_{i} / k T}, \quad \theta=\theta_{0}+\frac{1}{2} \theta_{2}-\frac{1}{2}, \\
K=\frac{1}{4}\left(\cosh ^{2} \lambda+2 \theta_{1} \cosh \lambda+\theta\right)^{-1}
\end{gathered}
$$

and $\lambda$ is to be determined from the not very transparent equation

$$
p=4 \mathrm{k} \sinh \lambda\left(\cosh \lambda+\theta_{1}\right) \equiv \frac{\tanh \lambda\left(1+\theta_{1} \operatorname{sech} \lambda\right)}{1+2 \theta_{1} \operatorname{sech} \lambda+\theta \operatorname{sech}^{2} \lambda} .
$$

Inserting these populations we find for the free energy

$$
\begin{aligned}
& A(p)=-N\left(\mu_{1} E p+\beta p^{2}+k T\left\{\ln \left[\left(1-p^{2}\right) / 4 K\right]\right.\right. \\
&+\left.\left.2 p\left(\tanh ^{-1} p-\lambda\right)\right\}\right)
\end{aligned}
$$

and for its derivatives

$$
\begin{aligned}
\partial A / \partial p & =-N\left\{\mu_{1} E+2 k T[(\beta p / k T)\right. \\
& \left.\left.+\tanh ^{-1} p-\lambda\right]\right\}, \\
\frac{\partial^{2} A}{\partial p^{2}} & =2 N k T\left[\frac{d \lambda}{d p}-\frac{1}{1-p^{2}}-\frac{\beta}{k T}\right]
\end{aligned}
$$

where $d \lambda / d p$ is determined by (1).

The equilibrium polarization is that which minimizes (2). This may occur for a completely polarized state $p=1$, or it may occur at a zero of (3a) with (3b) positive; i.e.,

$$
p=\tanh \left(\lambda-\frac{\beta p}{k T}-\frac{\mu_{1} E}{2 k T}\right)
$$

which must be solved simultaneously with (1). We note immediately that a solution $p=1$ cannot give a minimum in $A(p)$ if $\epsilon_{1}$ is finite. To show this we note that in all cases (1) shows that $\lambda \rightarrow \infty$ as $p \rightarrow 1$. Then from (1) we find that for $p \rightarrow 1$

$$
(1-p) /(1+p)=\theta_{1} e^{-\lambda}+(1+2 \theta) e^{-2 \lambda}+\cdots .
$$

Thus, for $\epsilon_{1}=\infty\left(\theta_{1}=0\right), \tanh ^{-1} p \rightarrow \lambda$, but for $\epsilon_{1}$ finite $\left(\theta_{1} \neq 0\right) \tanh ^{-1} p \rightarrow \lambda / 2$. Then from (3a), $\partial A / \partial p \rightarrow+\infty$ as $p \rightarrow 1$ if $\epsilon_{1}$ is finite. Thus, $A$ (1) cannot be a minimum.
In the unpolarized state $p=\lambda=E=0$, we have

$$
\begin{gathered}
A(0)=-N k T \ln \left(1+2 \theta_{1}+\theta\right), \\
S(0)=N k\left[\ln \left(1+2 \theta_{1}+\theta\right)\right. \\
\left.+\frac{\epsilon_{0}}{k T} \frac{\theta_{0}+2 \theta_{1}\left(\epsilon_{1} / \epsilon_{0}\right)+\frac{1}{2} \theta_{2}\left(\epsilon_{2} / \epsilon_{0}\right)}{1+2 \theta_{1}+\theta}\right] \\
\frac{\partial^{2} A}{\partial p^{2}}(0)=2 N k T\left(\frac{\theta+\theta_{1}}{1+\theta_{1}}-\frac{\beta}{k T}\right) .
\end{gathered}
$$

It is useful at this point to consider some special cases (at zero field):

(a) $\epsilon_{1}=\infty, \beta=0$ (this is Slater's original model). Equations (1) and (4) give only $p=\lambda=0$ (except at the critical temperature $\theta=1$, when any $p$ will do). The situation is indicated qualitatively in (a) of Fig. 2. The transition is 1st order with a jump to the completely ordered state. For $\epsilon_{2}=\infty, T_{c}=\epsilon_{0} /(k \ln 2)$ and just above $T_{c}$ (6) becomes $S_{c}=\frac{1}{2} N k \ln 2$.

(b) $\epsilon_{1}=\infty, \beta \neq 0$. The additional $-\beta p^{2}$ term gives the situation in (b) of the figure. The transition to $p=1$ occurs when $A(1)=-N \beta$ becomes equal to $A(0)$ given by (5), i.e., at a somewhat higher temperature and therefore higher entropy than in case (a).

(c) $\beta=0, \epsilon_{1}$ finite (this is Takagi's model). At high temperatures $p=\lambda=0$ is the only solution of (1) and (4); $A(0)$ is a minimum as indicated by the sign of (7). As $T$ is lowered till $\theta$ becomes less than $-\theta_{1},(7)$ changes sign giving a maximum in $A$ at $p=0$; Eqs. (1) and (4) then have another solution, namely $\operatorname{sech} \lambda$ $=-\theta_{1} / \theta$, corresponding to a minimum in $A$ which first appears at $p=0$ and moves to larger $p$ as $T$ is further reduced. The transition is second order as indicated in (c) of Fig. 2.

This conclusion is independent of the relative contributions of $\epsilon_{2}$ and $\epsilon_{0}$ to $\theta$ and thus contradicts the results of Grindlay and ter $\mathrm{Haar}^{5}$ who find first-order behavior for some cases of noninfinite $\epsilon_{2}$. The difficulty seems to be that they assign (in our notation) $\epsilon_{1}-\epsilon_{0} / 4$ to three, and $\epsilon_{1}+3 \epsilon_{0} / 4$ to one of the configurations marked with $\left(^{*}\right)$ in Fig. 1. We have repeated their calculation using equal energies and find a secondorder transition at $\theta=-\theta_{1}$ as expected.

(d) In the general case two qualitatively different behaviors are possible. The first is like that in (c) above. A nonzero solution of (1) and (4) first appears when (7) changes sign; that is, we have a second-order transition when

$$
\beta / k T=\left(\theta+\theta_{1}\right) /\left(1+\theta_{1}\right) .
$$

The second possibility corresponds to having two solutions of (1) and (4) in $0<p<1$ while there is still a

5 J. Grindlay and D. ter Haar, Proc. Roy, Soc. (London) A250, 266 (1959). 


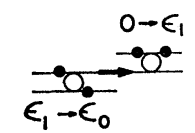

(a)

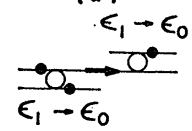

FIg. 4. Typical hydrogen jumps. In (a) the effect is to move an "ion" to the right. In (b) an "ion pair" is destroyed by recombination.

(b)

local minimum at $p=0$. There will then be a first-order transition at a temperature [greater than that given by (8)] when $A$, at the outer minimum, falls below $A(0)$. This is shown in (d) of Fig. 2.

The situation is summarized in Fig. 3. Each point in the figure represents a combination of parameters $\left(\theta, \theta_{1}\right.$, and $\left.\beta / k T\right)$ for which a transition takes place. In the upper left portion the transition is second order, and the figure simply represents equation (8). First order transitions occur in the lower right corner. The value of the polarization jump is indicated.

The susceptibility above $T_{c}$ is (for small $E$ )

$$
\chi=\frac{N^{2} \mu_{0} \mu_{1}}{V \partial^{2} A / \partial p^{2}}+\chi_{0}
$$

where the demoninator is given by (7) and vanishes for $T_{2}$, the root of (8), (not for $T_{c}>T_{2}$ in the first-order case). A Taylor expansion of (7) then gives Curie's law for temperatures not too far from the transition

$$
\chi=\left[C /\left(T-T_{2}\right)\right]+\chi_{0} .
$$

\section{EXPERIMENTAL EVIDENCE}

Let us now attempt to compare model and experiment. The model assumes one proton per bond. We note that diffusion of vacant (or doubly occupied) bonds would provide a mechanism for electrical conduction. Hydrogen motion indeed provides the mechanism, for Schmidt ${ }^{6}$ finds that hydrogen gas is evolved at the surface ( $\frac{1}{2}$ mole of $\mathrm{H}_{2}$ per Faraday) when charge is passed through the material. The temperature dependence of the conductivity yields an activation energy which may be associated with the energy of a vacancy and is so high, namely $0.58 \mathrm{eV}$, that the assumption of the model seems justified. ${ }^{7}$ Further evidence is provided by one of the mechanisms responsible for the relaxation of the deuterons in nuclear magnetic resonance experiments ${ }^{1}$ on $\mathrm{KD}_{2} \mathrm{PO}_{4}$. The angular dependence and dependence on magnetic quantum number identify the effect as that of the fluctuations of quadru-

6 V. H. Schmidt, Bull. Am. Phys. Soc. 7, 440 (1962).

${ }^{7}$ It has already been shown that the inclusion of empty and doubly occupied bonds would in any case introduce nothing essentially new insofar as the nature of the phase transition is concerned; see G. Shirane and T. Oguchi, J. Phys. Soc. Japan 3, 274 (1948). pole interaction produced by a jump between $x$ and $y$ bonds, i.e., by hydrogen diffusion. The activation energy is identical with that in the conductivity. ${ }^{1}$

The model assumes that the H's occupy off-center positions ordered at low temperatures and disordered at high temperatures. Below the transition this is shown by neutron diffraction. ${ }^{8}$ At high temperatures the diffraction results show a symmetrical distribution elongated along the bond, and the intrepretation of the model is only one of several which have been advanced. Some evidence is available in its favor, however. Jumps of deuterons from one off-center position to the other produce modulations of the quadrupole interactions which again contribute to magnetic relaxation but with characteristics quite distinct from the interbond jumping mentioned above. Such a process has been identified in the resonance experiments. The correlation time ${ }^{1}$ is about $10^{-11}$ sec at $215^{\circ} \mathrm{K}$ and varies as $\exp (0.078 \mathrm{eV} / k T)$; that is, the off-center positions do indeed have the relatively great lifetimes assumed by the model.

Furthermore, these experiments give a value of $\epsilon_{1}$ of the model. If low-energy $\left(0\right.$ or $\left.\epsilon_{0}\right)$ groups predominate, but a few of high energy $\left(\epsilon_{1}\right)$ are present, then the majority of along-the-bond jumps will be of the type indicated in Fig. 4 (a) in which neighboring low-energy and high-energy groups effectively interchange positions. The rate will go as the population, and hence the Boltzmann factor of the high-energy groups. Note that the jumps of Fig. 4(b) which destroy high-energy pairs by recombination will have a much smaller rate proportional to the population squared. The reverse pair-creation process must then be equally slow to maintain equilibrium; we expect, in fact, a factor $\exp \left(-2 \epsilon_{1} / k T\right)$ because the process requires energy approximately $2 \epsilon_{1}$ to create the pair.

For $\mathrm{KD}_{2} \mathrm{PO}_{4}$ then, we may identify the measured exponential in the jump rate described above with $\theta_{1}$ and take $\epsilon_{1} / k=900^{\circ} \mathrm{K}$.

One expects $\epsilon_{2}$, the energy of "doubly ionized" groups, to be approximately twice $\epsilon_{1}$ for "singly ionized" groups. If so, then the corresponding Boltzmann factor is negligibly small and we may as well set $\epsilon_{2}=\infty$.

For known $\epsilon_{1}$ and transition temperature, $T_{c}=213^{\circ} \mathrm{K},{ }^{9}$ Fig. 3 gives various possible combinations of $\epsilon_{0}$ and $\beta$; however, their ranges are not great: $\beta=0$ gives $\epsilon_{0} / k=154^{\circ} \mathrm{K}$, while $\beta / k=23^{\circ} \mathrm{K}$ (large enough for a first-order jump to $50 \%$ polarization) gives $\epsilon_{0} / k=110^{\circ} \mathrm{K}$. We may note that, although the transition is generally asserted to be second order, the smallest nonzero polarization reported ${ }^{10}$ is about $30 \%$. Spontaneous polarization curves using the measured $\epsilon_{1}$ are compared with experiment in Fig. 5 (a). Curve (3) with $\epsilon_{0} / k=116^{\circ} \mathrm{K}$

${ }^{8}$ R. S. Pease and G. E. Bacon, Proc. Roy. Soc. (London) A230, 359 (1955).

9 Subsequent to the calculations given in this paper, a higher value of $T_{c}$ for fully deuterated crystals has been reported, see Refs. 14 and 15.

${ }^{10}$ B. Zwicker and P. Scherrer, Helv. Phys. Acta 17, 346 (1944). 
Fig. 5. Comparison of theoretical spontaneous polarization with experiment (Ref. 10).
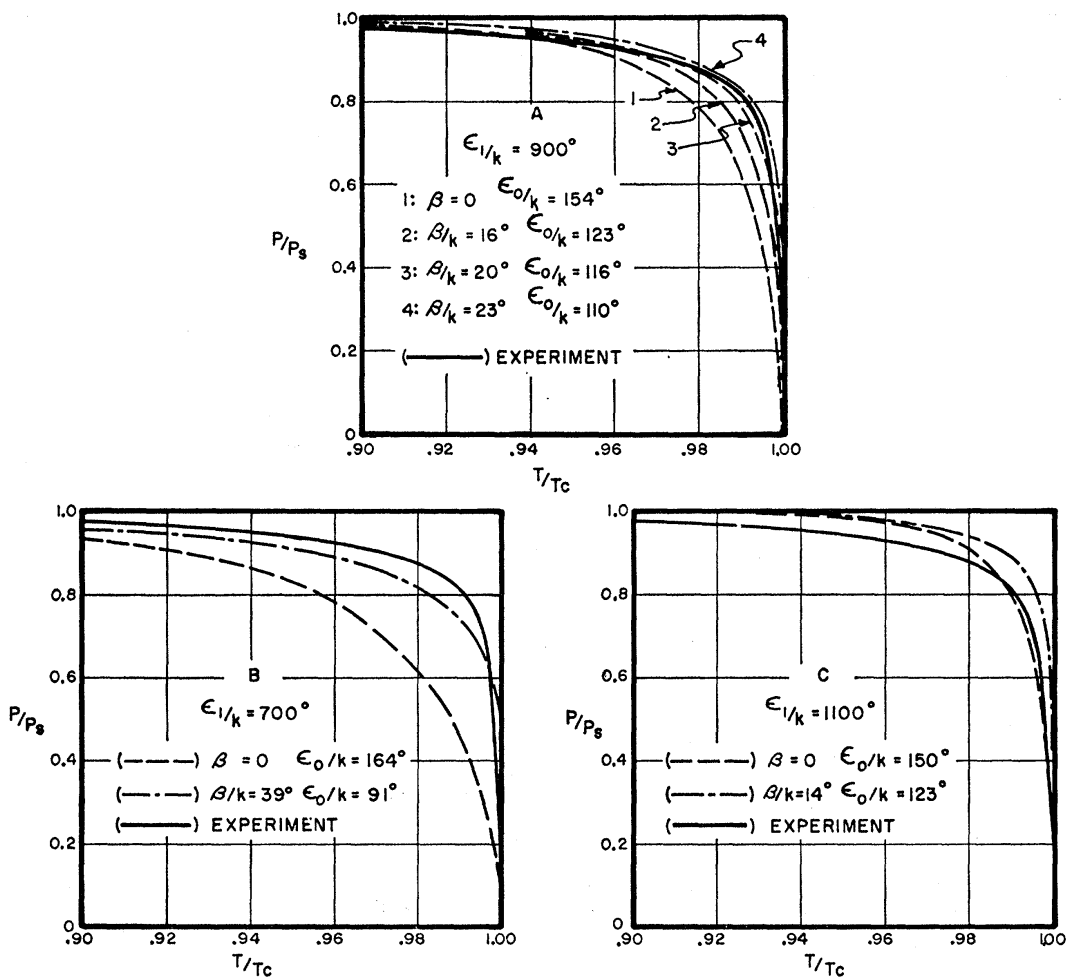

and $\beta / k=20^{\circ} \mathrm{K}$ (the maximum consistent with a second-order transition) will be taken, somewhat arbitrarily, as giving the best fit.

Figure 5 (b) and (c) show the effect of trying to depart from the measured $\epsilon_{1}$ by $\pm 20 \%$. Curves which bracket the experimental behavior near $T_{c}$ are found at lower temperatures to run too high in one case and too low in the other.

In short, the energy parameters of the model can be fitted essentially uniquely and the $\epsilon_{1}$ value required to fit the observed polarization is just the value obtained from quite independent measurements.

To predict the susceptibility from (9) we need values of $\mu_{0}$ and $\chi_{0}$. Mayer's value ${ }^{11}$ for the saturated spontaneous polarization, $P_{\text {sat }}=4.5 \times 10^{-6} \mathrm{C} / \mathrm{cm}^{2}=N \mu_{0} / V$, yields $\mu_{0}=0.27$ e $\AA=1.3 \times 10^{-18}$ esu. $\chi_{0}$ can be estimated at 0.5 by fitting (10) to the high-temperature susceptibility ${ }^{12}$ of $\mathrm{KH}_{2} \mathrm{PO}_{4}$ and assuming no change on deuteration. These values give $C=163^{\circ} \mathrm{K}$ for the Curie constant in (10) as a prediction of the model, while the experimental values ${ }^{11,13-15}$ range from $210^{\circ}$ to $330^{\circ}$. We regard the agreement as gratifying in view of the simplicity of the assumptions. (The model could be made to fit by varying $\chi_{0}: \chi_{0}=0$ yields $C=141^{\circ}$ and

${ }^{11}$ R. J. Mayer, M. S. thesis, Department of Electrical Engineering, University of Washington, Seattle, Washington, 1961 R. J. Mayer and J. L. Bjorkstam, J. Phys. Chem. Solids 23, 619 (1962).

${ }_{12}$ G. Busch, Helv. Phys. Acta 11, 269 (1938).

${ }^{13} \mathrm{~W}$. Bantle, Helv. Phys. Acta 15, 373 (1942)

${ }_{14}$ R. M. Hill and S. K. Ichiki, Phys. Rev. 150, (1963)

${ }^{15}$ T. R. Sliker and S. R. Burlage, J. Appl. Phys. 34, 1837 (1963). $\chi_{0}=5$ yields $C=365^{\circ}$. In the last case, however, $\chi^{-1}$ would be markedly nonlinear in $T$.)

The heat capacity predicted by the model is compared with experiment ${ }^{13}$ in Fig. 6 . There is, of course, the problem of subtracting the phonon heat capacity from the experimental values; the two curves represent the same data, first with a constant and second with a linear function of temperature subtracted. We note that the qualitative agreement with experiment is markedly better for the model with finite $\beta$ than for $\beta=0$.

However, there is quantitative disagreement for the area under the curve. The expression (6), for the entropy at the transition conditions of Fig. 3, seems inevitably to give more than the observed value, as is indicated in Table I. Even the extreme Slater limit

TABLE I. Transition entropy.

\begin{tabular}{|c|c|c|}
\hline & & $S / R$ \\
\hline \multicolumn{2}{|c|}{$\begin{array}{l}\text { Experiment } \\
\text { With constant } C_{\text {lattice }} \text { subtracted } \\
\text { With linear } C_{\text {lattice }} \text { subtracted }\end{array}$} & $\begin{array}{l}0.24 \\
0.32\end{array}$ \\
\hline $\begin{array}{l}\text { Theory } \\
\epsilon_{1} / k\left({ }^{\circ} \mathrm{K}\right)\end{array}$ & $\beta / k\left({ }^{\circ} \mathrm{K}\right)$ & \\
\hline \multirow[t]{2}{*}{900} & 20 (best fit) & $\begin{array}{l}0.50 \\
0.48\end{array}$ \\
\hline & 23 (first order, $50 \%$ jump) & 0.50 \\
\hline \multirow{3}{*}{1100} & 0 & 0.62 \\
\hline & 0 & 0.41 \\
\hline & 12 (maximum for second order) & 0.43 \\
\hline$\infty$ & 0 (Slater) & 0.35 \\
\hline
\end{tabular}




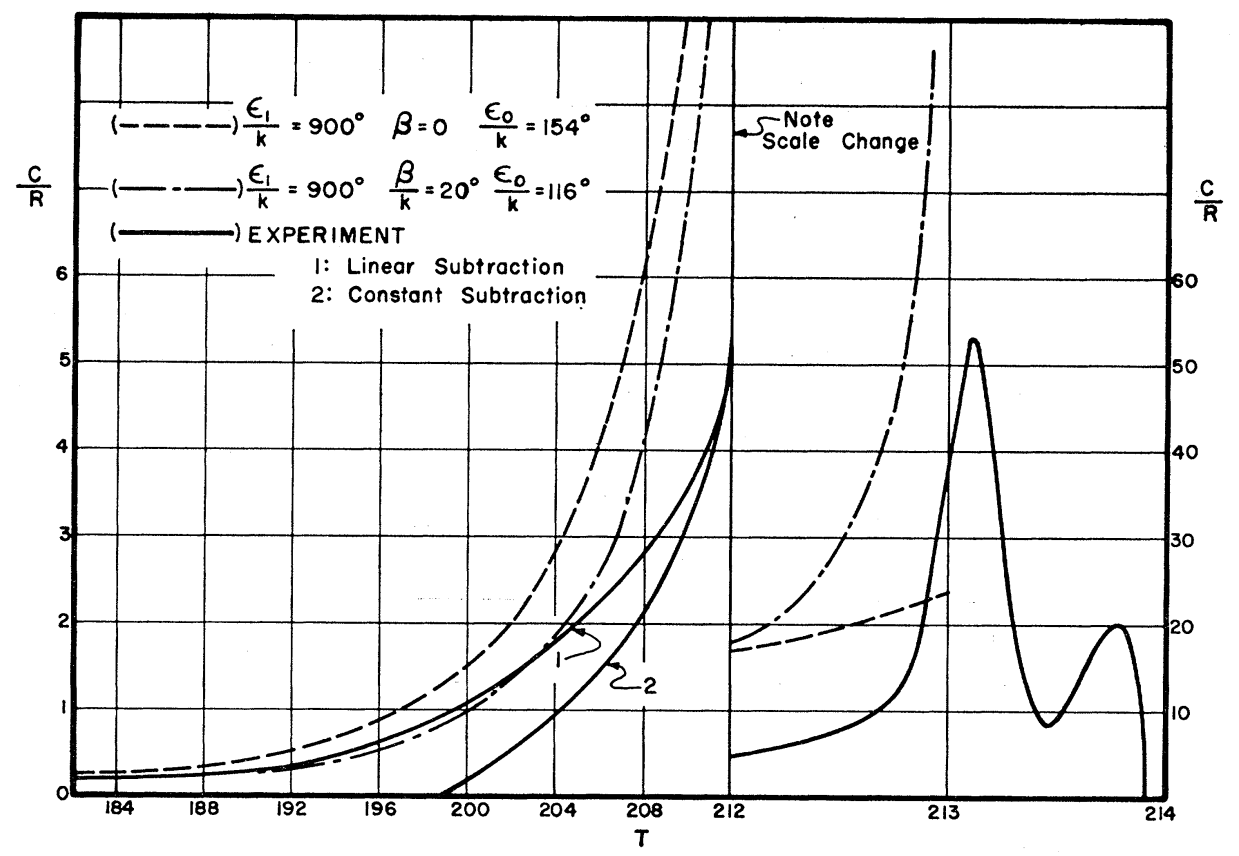

Fig. 6. Comparison of theoretical specific heat with experiment (Ref. 13).

with high-energy groups completely forbidden gives somewhat too much entropy.

\section{TENTATIVE SUGGESTIONS WITH REGARD TO DISCREPANCIES}

The small experimental entropy suggest that shortrange ordering of the hydrogens persists above $T_{c}$ to an extent not predicted by the model. This is perhaps not surprising. Although the hydrogen motions are very nearly along $x$ or $y$, the model concerns itself only with moment contributions along $z$, the direction in which the spontaneous polarization appears, so that correlations to which the assignments of Fig. 1 are indifferent may in fact be important. Such correlations have been postulated in a model ${ }^{16}$ for the antiferroelectric behavior of $\left(\mathrm{NH}_{4}\right) \mathrm{H}_{2} \mathrm{PO}_{4}$.

Some support for this view appears if one attempts to use the $\mathrm{KD}_{2} \mathrm{PO}_{4}$ parameters to predict those for the

${ }^{16}$ T. Nagamiya, Prog. Theoret. Phys. (Japan) 7, 275 (1952). undeuterated crystal. Since the configurations of Fig. 1 become identical for hydrogens centered on their bonds one is tempted to assume that the energies $\epsilon$ for different particles vary as the square of the off-center distance. This distance is greater for deuterons than it is for protons (as one would expect if the deuteron lies lower in an unsymmetric well ${ }^{17}$ ) and in fact leads to estimates for the $\epsilon$ 's of $\mathrm{KH}_{2} \mathrm{PO}_{4}$ enough lower to account moderately well for its lower $T_{c}\left(122^{\circ} \mathrm{K}\right)$. If one supposes that $\beta$, which measures the long-range effects, depends only on the $z$ moments, one would expect it to vary as the square of the saturated polarization which is nearly the same for the two isomorphs. Figure 3 then indicates that the transition point for $\mathrm{KH}_{2} \mathrm{PO}_{4}$ would. lie well within the first-order region, whereas in fact the spontaneous polarization rises less rapidly than in the deuterated case. The difficulty disappears if the smaller $x$ and $y$ motions in $\mathrm{KH}_{2} \mathrm{PO}_{4}$ are considered to give a smaller $\beta$.

${ }^{17}$ C. Reid, J. Chem. Phys. 30, 182 (1959). 